\title{
Transbronchial Lung Cryobiopsy is Safe and Effective for Diagnosing Acutely III Hospitalized Patients with New Diffuse Parenchymal Lung Disease
}

\author{
Carson Castellani ${ }^{1} \cdot$ Henry Castellani ${ }^{2} \cdot$ Bryan S. Benn $^{3}$
}

Received: 1 December 2021 / Accepted: 8 January 2022 / Published online: 1 February 2022

(c) The Author(s), under exclusive licence to Springer Science+Business Media, LLC, part of Springer Nature 2022

\begin{abstract}
Introduction Transbronchial lung cryobiopsy (TBLC) is an accepted alternative to surgical lung biopsy (SLB) for diagnosing diffuse parenchymal lung disease (DPLD) that is less invasive and results in comparable diagnostic yields. Performing lung biopsies on hospitalized patients, however, has increased risk due to the patient's underlying disease severity. Data evaluating the safety and efficacy of TBLC in hospitalized patients are limited. We present a comparison of TBLC for hospitalized and outpatients and provide the safety and diagnostic yields in these populations.

Methods Demographic data, pulmonary function values, chest imaging pattern, procedural information, and diagnosis were recorded from enrolled patients. Complications from the procedure were the primary outcomes and diagnostic yield was the secondary outcome.

Results 77 patients ( $n=22$ hospitalized vs $n=55$ outpatient) underwent TBLC during the study period. Comparing adverse events between hospitalized and outpatients revealed no statistically significant differences in pneumothorax ( $9 \%, n=2$ vs $5 \%, n=3)$, tube thoracostomy placement $(5 \%, n=1$ vs $2 \%, n=1)$, grade 2 bleeding $(9 \%, n=2$ vs $0 \%, n=0)$, escalation in level of care (5\%,n=1 vs $0 \%, n=0)$, 30-day mortality ( $9 \%, n=2$ vs $2 \%, n=1)$, and 60 -day mortality $(9 \%, n=2$ vs $4 \%, n=2)$ $(p>0.05$ for all). No deaths were attributed to the procedure. $95 \%$ of cases received a multidisciplinary conference diagnosis (hospitalized $100 \%, n=22$ vs outpatients $93 \%, n=51, p=0.32$ ).

Conclusion Our experience supports that TBLC may be a safe and effective modality for acutely ill-hospitalized patients with DPLD. Further efforts to enhance procedural safety and to determine the impact of an expedited tissue diagnosis on patient outcomes are needed.
\end{abstract}

Keywords Diffuse parenchymal lung disease $\cdot$ Interstitial lung disease $\cdot$ Transbronchial lung cryobiopsy $\cdot$ Respiratory failure

\section{Abbreviations}

ASA American Society of Anesthesiologists

DPLD Diffuse parenchymal lung disease

REBUS Radial endobronchial ultrasound

IP Interventional pulmonology

Bryan S. Benn

Bbenn@mcw.edu

1 Department of Medicine, Medical College of Wisconsin, Milwaukee, WI, USA

2 Wayne State University School of Medicine, Detroit, MI, USA

3 Division of Pulmonary and Critical Care, Department of Medicine, Medical College of Wisconsin, $8701 \mathrm{~W}$ Watertown Plank, Milwaukee, WI 53226, USA
IPF Idiopathic pulmonary fibrosis

HRCT High-resolution CT chest

MDC Multidisciplinary conference

SLB Surgical lung biopsy

TBLC Transbronchial lung cryobiopsy

UIP Usual interstitial pneumonia

\section{Introduction}

Diagnosing diffuse parenchymal lung disease (DPLD) remains challenging. A multidisciplinary conference (MDC) is recommended to evaluate patient factors, radiographic findings, and often histopathological data [1]. Guidelines recommend obtaining a tissue biopsy for suspected DPLD when there is diagnostic uncertainty based on non-invasive 
data alone [1]. Options available for obtaining a tissue sample include transbronchial lung cryobiopsy (TBLC) and surgical lung biopsy (SLB), which has traditionally been the standard method. When TBLC is used in conjunction with an MDC, it represents a reasonable alternative to SLB with comparable accuracy, decreased morbidity, and potential cost savings [2-5].

While lung biopsy is generally considered safe and effective, studies have suggested that hospitalized patients who undergo this procedure might be at higher risk of complications compared to non-hospitalized patients. Performing SLB on hospitalized patients with suspected DPLD results in high rates of complication with significant morbidity and mortality rates as high as $16 \%$ [6-9].

There are limited data evaluating whether TBLC is a safe and effective modality to obtain a tissue sample for hospitalized patients with suspected DPLD. A prior study revealed that hospitalized patients undergoing TBLC have an objectively higher level of acuity compared to outpatients in terms of supplemental oxygen requirement, comorbidities requiring steroid, immunosuppressive or antibiotic treatment, and American Society of Anesthesiologists (ASA) scores [10]. To help determine whether TBLC is safe and effective for acutely ill-hospitalized patients with suspected DPLD we present a comparison of TBLC procedures performed at our institution for hospitalized and outpatients and provide the safety and diagnostic yields in these populations.

\section{Methods}

\section{Study Cohort}

All patients consecutively presenting to our interventional pulmonology (IP) clinic or inpatient consult service for evaluation of newly discovered DPLD by high-resolution CT chest (HRCT) from November 2019 through May 2021 were consented for TBLC and enrolled. Patients with DPLD that were already on treatment (immunosuppressive or antifibrotic therapy prescribed specifically for lung disease), were unable to stop anti-platelet or anti-thrombotic medications, or had a platelet value $<50 \times 10^{9} / \mathrm{L}$ were excluded. Pulmonary hypertension was evaluated prior to procedure by transthoracic echocardiogram, if indicated. Decision to proceed with biopsy was made in collaboration with the MDC when able. The study was approved by the institutional review board of the Medical College of Wisconsin PRO00036023.

Demographic data, pulmonary function test (PFT) values, chest imaging pattern, which was determined as per established criteria [1], medications, comorbidities, and supplemental oxygen need were extracted from the medical record. Procedural information, including biopsy location, number of lobes biopsied, total biopsies performed, and final MDC diagnosis, were recorded. Complications from the procedure, including pneumothorax, bleeding severity, worsening respiratory status, change in level of care, and 30-day and 60-day mortality, were the primary outcomes. Diagnostic yield from the procedure was the secondary outcome.

\section{Procedure Description}

All procedures were performed under general anesthesia with neuromuscular blockade and positive end expiratory pressure of 5-10 $\mathrm{cm} \mathrm{H}_{2} \mathrm{O}$ through an 8.5-mm endotracheal tube. A flexible bronchoscope (Olympus BF-1TH190, inner diameter $2.8 \mathrm{~mm}$ or Olympus BF-XT190, inner diameter $3.2 \mathrm{~mm}$, Olympus America, Center Valley, PA, USA) was used for airway inspection. Bronchoalveolar lavage was then performed to evaluate for infectious process as per guideline recommendations [11].

Based on imaging review, the bronchoscope was then directed to targeted abnormal areas. $3 \mathrm{ml}$ of phenylephrine (1:100,000 dilution from a stock $100 \mathrm{mcg} / \mathrm{ml}$ concentration) was instilled into each identified airway segment and allowed to dwell for $\sim 3$ min to promote vasoconstriction and to mitigate bleeding risk. Under fluoroscopic guidance, a transbronchial forceps biopsy was performed to evaluate for bleeding tendency in the periphery of the lung beyond the area visualized. Additional biopsies were performed for microbiology studies and the Envisia Genomic Classifier, if clinically indicated.

A disposable (1.7 mm, 31 cases, $\mathrm{CO}_{2}$ as the cryogen) or reusable (1.9 mm, 46 cases, $\mathrm{N}_{2} \mathrm{O}$ as the cryogen) cryoprobe (Erbe Medizintechnik GmbH, Tübingen, Germany) was used for all procedures. Prior to biopsy, the cryoprobe was tested in room-temperature saline to determine optimal activation time. The cryoprobe was then inserted through the working channel, directed under fluoroscopic guidance to the target area, advanced until resistance was met, and then pulled back to within $0.5-1.0 \mathrm{~cm}$ of the pleura.

While the patient was apneic, the cryoprobe was activated under fluoroscopic guidance and the bronchoscope and cryoprobe were removed en bloc. After the specimen was thawed in room-temperature saline, the cryoprobe was removed from the working channel. The bronchoscope was then reinserted to evaluate for bleeding with ventilation resumed. Once biopsies were complete and airway hemostasis was confirmed, a post-procedure chest x-ray was performed immediately after the procedure to evaluate for pneumothorax. For 9 patients (8 outpatient and 1 inpatient), TBLC was performed with cone-beam CT guidance as previously described [12]. 


\section{Statistical Analysis}

Categorical variables were expressed as a number and percentage and comparisons were done with the Chi-square test or the Fisher's exact test. Continuous variables were expressed as mean with standard deviation or as median with interquartile range and comparison were done with the student's t test. All p-values were two-sided and $p<0.05$ was considered statistically significant. Statistical analysis was performed with SAS version 9.4 (SAS Institute).

\section{Results}

77 patients ( $n=22$ hospitalized vs $n=55$ outpatient) underwent TBLC during the study period. There were no significant differences in age, sex, race/ethnicity, smoking status, PFTs, or chest imaging pattern between the groups (Table 1). However, inpatients had a statistically significant increased ASA score $(3.3 \pm 0.48)$ compared to outpatients $(3.0 \pm 0.51)(p=0.0062)$. Additionally, inpatients had more total comorbidities on average than outpatients $(2.9 \pm 1.6 \mathrm{vs}$ $1.9 \pm 1.2)(p=0.0070)$ and were more likely to carry certain comorbidities, including history of malignancy $(68 \%$ $n=15$ vs $24 \% n=13)(p=0.0002)$ or history of transplant $(31 \% n=7$ vs $4 \% n=2)(p=0.0017)$ (Table 2). Hospitalized patients were more likely to be receiving treatment with steroids for non-pulmonary disease ( $45 \% n=10$ vs $18 \% n=10)$ $(p=0.0137)$, were more likely to be receiving immunosuppressive medication $(36 \% n=8$ vs $13 \% n=7)(p=0.0266)$, and were more likely to require supplemental oxygen $(55 \%$ $n=12$ vs $18 \% n=10$ ). Admission diagnoses for hospitalized patients included hypoxic respiratory failure $(n=11)$, COVID-19 pneumonia $(n=2)$, dyspnea $(n=2)$, interstitial lung disease $(n=2)$, altered mental status $(n=1)$, cholecystitis $(n=1)$, pneumonia $(n=1)$, pulmonary nodules $(n=1)$, and severe sepsis $(n=1)$.

TBLC was done in a single lobe in $39(51 \%)$ patients, while $38(49 \%)$ had two lobes sampled (Table 3$)$. The average number of biopsies per procedure was $4.4 \pm 1.5$. The right lower lobe was most frequently biopsied at $53 \%$ followed by the right upper lobe $30 \%$, the left lower lobe at $9 \%$, and the left upper lobe at $8 \%$.

Complications from the procedure were similar in both groups (Table 4). Pneumothorax occurred in $6 \%(n=5)$ of patients ( $n=2,9 \%$ inpatient vs $n=3,5 \%$ outpatient) $(p=0.6202)$. Chest tube placement was required in $3 \%$ $(n=2)$ of patients ( $n=1,5 \%$ inpatient vs $n=1,2 \%$ outpatient) $(p=0.4925)$. All chest tubes were removed within $24 \mathrm{~h}$. Grade 2 bleeding, as per consensus definition [13], occurred in $3 \%(n=2)$ of patients, both of whom were inpatients ( $n=2,9 \%$ inpatient), and of which one, although hemodynamically stable, was transferred to the intensive
Table 1 Patient Characteristics

\begin{tabular}{|c|c|c|c|c|}
\hline & Outpatient & Inpatient & Total & $\mathrm{p}$ value \\
\hline Age $($ mean $\pm S D)$ & $64 \pm 13$ & $61 \pm 10$ & $63 \pm 12$ & 0.3191 \\
\hline $\operatorname{Sex}(\mathrm{n}, \%)$ & 55 & 22 & 77 & 0.1494 \\
\hline Female & $30(55 \%)$ & $8(36 \%)$ & $38(49 \%)$ & \\
\hline Male & $25(45 \%)$ & $14(64 \%)$ & $39(51 \%)$ & \\
\hline Race (n, \%) & & & & 0.3126 \\
\hline Caucasian & $43(78 \%)$ & $16(72 \%)$ & $59(77 \%)$ & \\
\hline Black & $7(13 \%)$ & $3(14 \%)$ & $10(13 \%)$ & \\
\hline Hispanic & $2(4 \%)$ & $3(14 \%)$ & $5(6 \%)$ & \\
\hline Asian & $3(5 \%)$ & $0(0 \%)$ & $3(4 \%)$ & \\
\hline $\mathrm{BMI}($ mean $\pm \mathrm{SD})$ & $31 \pm 8$ & $30 \pm 7$ & $31 \pm 8$ & 0.4618 \\
\hline $\mathrm{BMI}<30(\mathrm{n}, \%)$ & $29(53 \%)$ & $13(59 \%)$ & $42(55 \%)$ & \\
\hline $\mathrm{BMI}>30(\mathrm{n}, \%)$ & $26(47 \%)$ & $9(41 \%)$ & $35(45 \%)$ & \\
\hline Smoking Status (n, \%) & & & & 0.9106 \\
\hline Current & $2(4 \%)$ & $1(4 \%)$ & $3(4 \%)$ & \\
\hline Former & $28(51 \%)$ & $12(55 \%)$ & $40(52 \%)$ & \\
\hline Never & $25(45 \%)$ & $9(41 \%)$ & $34(44 \%)$ & \\
\hline $\begin{array}{l}\text { ASA Score } \\
\quad(\text { mean } \pm \text { SD })\end{array}$ & $3.0 \pm 0.51$ & $3.3 \pm 0.48$ & $3.1 \pm 0.52$ & 0.0062 \\
\hline \multicolumn{5}{|l|}{ PFTs $($ mean $\pm S D)$} \\
\hline FEV $1 \%$ predicted & $71 \pm 20$ & $69 \pm 19$ & $71 \pm 20$ & 0.8154 \\
\hline FVC $\%$ predicted & $68 \pm 17$ & $66 \pm 19$ & $68 \pm 17$ & 0.6194 \\
\hline TLC $\%$ predicted & $79 \pm 22$ & $83 \pm 19$ & $80 \pm 22$ & 0.5372 \\
\hline DLCO $\%$ predicted & $60 \pm 19$ & $62 \pm 29$ & $60 \pm 21$ & 0.8626 \\
\hline HRCT Pattern (n, \%) & & & & 0.5565 \\
\hline Alternative & $35(64 \%)$ & $19(85 \%)$ & $54(70 \%)$ & \\
\hline Probable UIP & $4(7 \%)$ & $1(5 \%)$ & $5(7 \%)$ & \\
\hline Indeterminant for UIP & $3(6 \%)$ & $1(5 \%)$ & $4(5 \%)$ & \\
\hline NSIP & $5(9 \%)$ & $0(0 \%)$ & $5(7 \%)$ & \\
\hline HP & $7(12 \%)$ & $1(5 \%)$ & $8(10 \%)$ & \\
\hline Pneumonitis & $1(2 \%)$ & $0(0 \%)$ & $1(1 \%)$ & \\
\hline
\end{tabular}

Bold value indicates statistical significance $(\mathrm{p}<0.05)$

Data are expressed as number (\%) or mean \pm standard deviation

ASA American Society of Anesthesiologists, DLCO diffusing capacity for carbon monoxide, $F E V 1$ forced expiratory volume in $1 \mathrm{sec}$ ond, $F V C$ forced vital capacity, $H P$ hypersensitivity pneumonitis, $H R C T$ high-resolution CT chest, NSIP nonspecific interstitial pneumonitis, $S D$ standard deviation, $T L C$ total lung capacity, UIP usual interstitial pneumonia

care unit for closer monitoring as per the primary care team's decision ( $n=1,2 \%$ of patients, $5 \%$ of inpatients, transfer in level of care). For all other patients, only grade 1 bleeding occurred, which did not require any intervention aside from brief suctioning with observation until hemostasis was confirmed. All cause 30-day and 60-day mortality were similar between the two groups and no deaths were directly attributable to the procedure. For the inpatient mortalities, one patient died ten days after the procedure, which was one week after discharge from the hospital, due to out of hospital cardiac arrest with ventricular fibrillation on initial 
Table 2 Comorbidities and medications

\begin{tabular}{|c|c|c|c|c|}
\hline & $\begin{array}{l}\text { Outpatient } \\
n(\%)\end{array}$ & $\begin{array}{l}\text { Inpatient } \\
n(\%)\end{array}$ & $\begin{array}{l}\text { Total } \\
n(\%)\end{array}$ & $p$ value \\
\hline \multicolumn{5}{|l|}{ Comorbidities } \\
\hline Hypertension & $32(58 \%)$ & $16(73 \%)$ & $48(62 \%)$ & 0.234 \\
\hline GERD & $24(44 \%)$ & $7(32 \%)$ & $31(40 \%)$ & 0.3394 \\
\hline Malignancy & $13(24 \%)$ & $15(68 \%)$ & $28(36 \%)$ & 0.0002 \\
\hline Diabetes & $15(27 \%)$ & $8(36 \%)$ & $23(30 \%)$ & 0.4311 \\
\hline Cardiac (CAD or CHF) & $9(16 \%)$ & $6(27 \%)$ & $15(19 \%)$ & 0.3421 \\
\hline a Post-Transplant & $2(4 \%)$ & $7(31 \%)$ & $9(12 \%)$ & 0.0017 \\
\hline OSA & $11(20 \%)$ & $4(18 \%)$ & $15(19 \%)$ & 1 \\
\hline Any Comorbidity & $48(87 \%)$ & $20(91 \%)$ & $68(88 \%)$ & 1 \\
\hline Comorbidity Count $($ mean \pm SD) & $1.9 \pm 1.2$ & $2.9 \pm 1.6$ & $2.2 \pm 1.4$ & 0.00701 \\
\hline \multicolumn{5}{|l|}{ Medications } \\
\hline Aspirin & $15(27 \%)$ & $7(32 \%)$ & $22(29 \%)$ & 0.69 \\
\hline $\mathrm{P} 2 \mathrm{Y} 12$ & $6(11 \%)$ & $0(0 \%)$ & $6(8 \%)$ & 0.1745 \\
\hline Anticoagulation & $8(15 \%)$ & $3(14 \%)$ & $11(14 \%)$ & 1 \\
\hline Steroids & $10(18 \%)$ & $10(45 \%)$ & $20(26 \%)$ & 0.0137 \\
\hline${ }^{\mathrm{b}}$ Immunosuppressives & $7(13 \%)^{1}$ & $8(36 \%)^{2}$ & $15(19 \%)^{1}$ & 0.02661 \\
\hline Oxygen Requirement & & & & 0.0013 \\
\hline Room Air & $45(82 \%)$ & $10(45 \%)$ & $55(71 \%)$ & \\
\hline Nasal Cannula & $10(18 \%)$ & $9(41 \%)$ & $19(25 \%)$ & \\
\hline HFNC & $0(0 \%)$ & $3(14 \%)$ & $3(4 \%)$ & \\
\hline
\end{tabular}

Bold values indicate statistical significance $(\mathrm{p}<0.05)$

Data are expressed as number (\%)

$C A D$ coronary artery disease, $C H F$ congestive heart failure, GERD gastroesophageal reflux disease, $H F N C$ high-flow nasal cannula, $O S A$ obstructive sleep apnea, $S D$ standard deviation

aPost-transplant organs include bone marrow $(n=5)$, liver $(n=2)$, and kidney $(n=2)$

${ }^{\mathrm{b}}$ Immunosuppressives include chemotherapy $(n=7)$, immunomodulatory $(n=4)$, and anti-rejection medications $(n=6) .2$ patients were on multiple classes of immunosuppressive medications
Table 3 Procedural characteristics

\begin{tabular}{llll}
\hline & $\begin{array}{l}\text { Outpatient } \\
n(\%)\end{array}$ & $\begin{array}{l}\text { Inpatient } \\
n(\%)\end{array}$ & $\begin{array}{l}\text { Total } \\
n(\%)\end{array}$ \\
\hline $\begin{array}{l}\text { Biopsy Information } \\
\text { One Lobe }\end{array}$ & $27(49 \%)$ & $12(55 \%)$ & $39(51 \%)$ \\
Two Lobes & $28(51 \%)$ & $10(45 \%)$ & $38(49 \%)$ \\
$\quad$ Total Biopsies (mean \pm SD) & $4.5 \pm 1.5$ & $4.3 \pm 1.5$ & $4.4 \pm 1.5$ \\
Location & & & \\
Left Upper Lobe & $7(8 \%)$ & $3(9 \%)$ & $9(8 \%)$ \\
Left Lower Lobe & $6(7 \%)$ & $3(9 \%)$ & $10(9 \%)$ \\
Right Upper Lobe & $27(33 \%)$ & $8(26 \%)$ & $35(30 \%)$ \\
Right Lower Lobe & $43(52 \%)$ & $18(56 \%)$ & $61(53 \%)$ \\
\hline
\end{tabular}

Data are expressed as number $(\%)$ or mean \pm standard deviation $S D$ standard deviation

rhythm. The other patient died on hospice due to refractory
Table 4 Adverse events

\begin{tabular}{lllll}
\hline & $\begin{array}{l}\text { Outpatient } \\
n(\%)\end{array}$ & $\begin{array}{l}\text { Inpatient } \\
n(\%)\end{array}$ & $\begin{array}{l}\text { Total } \\
n(\%)\end{array}$ & $p$ value \\
\hline Pneumothorax & $3(5)$ & $2(9)$ & $5(6)$ & 0.6202 \\
Chest Tube & $1(2)$ & $1(5)$ & $2(3)$ & 0.4925 \\
Grade II Bleeding & $0(0)$ & $2(9)$ & $2(3)$ & 0.0789 \\
Transfer in Level of Care & $0(0)$ & $1(5)$ & $1(1)$ & 1 \\
All Cause 30-day Mortality & $1(2)$ & $2(9)$ & $3(4)$ & 0.1947 \\
All Cause 60-day Mortality & $2(4)$ & $2(9)$ & $4(5)$ & 0.5735 \\
\hline
\end{tabular}

Data are expressed as number (\%)

hypoxemic respiratory failure due to ARDS from COVID-19 pneumonia 29 days after the procedure.

A final MDC diagnosis was obtained in $95 \%(n=73)$ of patients (inpatient $100 \% n=22$ vs outpatients $93 \%$ $n=51)(p=0.32)$ (Table 5). The most common diagnosis was chronic hypersensitivity pneumonitis $(26 \%, n=20)$, 
Table 5 Biopsy results

\begin{tabular}{lllll}
\hline & $\begin{array}{l}\text { Outpatient } \\
n(\%)\end{array}$ & $\begin{array}{l}\text { Inpatient } \\
n(\%)\end{array}$ & $\begin{array}{l}\text { Total } \\
n(\%)\end{array}$ & $p$ value \\
\hline MDC Diagnosis & $51(93)$ & $22(100)$ & $73(95$ & \\
Chronic HP & $19(35)$ & $1(5)$ & $20(26)$ \\
CTD-ILD & $10(18)$ & $3(14)$ & $13(17)$ & \\
Organizing Pneumonia & $2(4)$ & $5(23)$ & $7(9$ \\
Drug-Induced Pneumo- & $2(4)$ & $3(14)$ & $5(7)$ & \\
$\quad$ nitis & & & & \\
Granulomatous Pneumo- & $4(7)$ & $0(0)$ & $4(5)$ \\
$\quad$ nitis & & & & \\
Unclassifiable ILD & $4(7)$ & $0(0)$ & $4(5)$ \\
CFILD & $0(0)$ & $2(9)$ & $2(3)$ \\
IPF & $3(5)$ & $1(5)$ & $4(5)$ \\
Vaping-Induced Lung & $1(2)$ & $1(5)$ & $2(3)$ & \\
$\quad$ Injury & & & & \\
Lung Adenocarcinoma & $1(2)$ & $1(5)$ & $2(3)$ \\
Bronchiolitis & $1(2)$ & $1(5)$ & $2(3)$ \\
Emphysema & $2(4)$ & $0(0)$ & $2(3)$ \\
aOther & $6(10)$ & $4(15)$ & $10(11)$ & 0.3200 \\
\hline
\end{tabular}

CFILD chronic fibrosing interstitial lung disease, CTD-ILD connective tissue disease-related interstitial lung disease, $H P$ hypersensitivity pneumonitis, ILD interstitial lung disease, IPF idiopathic pulmonary fibrosis

${ }^{\text {a }}$ Other includes bronchiolitis $(n=1)$, CMV pneumonitis $(n=1)$, combined pulmonary fibrosis and emphysema $(n=1)$, granulomatous and lymphocytic interstitial lung disease $(n=1)$, graft versus host disease $(n=1)$, interstitial pneumonia with autoimmune features $(n=1)$, myelofibrosis $(n=1)$, plastic bronchitis $(n=1)$, pneumonia $(n=1)$, and respiratory bronchiolitis interstitial lung disease $(n=1)$

followed by connective tissue disease-related interstitial lung disease $(17 \%, n=13)$, organizing pneumonia (9\%, $n=7)$, and drug-induced pneumonitis $(7 \%, n=5)$. Four (5\%) patients (all outpatients) were determined to have unclassifiable DPLD and did not receive a definitive diagnosis.

\section{Discussion}

While the utility of TBLC in establishing a diagnosis for patients with DPLD is well established, concerns remain regarding potential complications, including pneumothorax and bleeding. Debate also centers upon optimal patient selection. We present our analysis comparing complication rates in outpatients and inpatients to better assess the safety profile of TBLC in different patient populations. Although inpatients had an objectively higher degree of clinical acuity, as indicated by statistically significant differences in comorbidities (history of malignancy, history of transplant, supplemental oxygen requirement), and ASA scores (Tables 1 and 2), rates of complications and the ability to obtain a diagnosis were similar.

Despite various procedural techniques employed to mitigate against complications developing, ensuring safety during TBLC remains a challenge due to the increased risk of pneumothorax and bleeding compared to conventional forceps biopsy. Recent meta-analyses of TLBC procedures reported pneumothorax rates of $9.5-12 \%$ and moderate to severe, or grade 2 bleeding, of $4.9-39 \%$ [14, 15]. Our study revealed a comparable incidence of pneumothorax $(6 \%, n=5$, of patients; $9 \%, n=2$, inpatients; $5 \%, n=3$, outpatients) and grade 2 bleeding (3\%, $n=2$, of patients; $9 \%$, $n=2$, inpatients; $0 \%, n=0$ outpatients) compared to prior studies with no differences seen in the more acutely ill inpatient group (Table 4). These results are also comparable to the prior study comparing inpatients to outpatients, in which no statistically significant difference in complication rates was found [10].

Best practice recommendations based on expert guidelines exist for TBLC. These include using a large diameter endotracheal tube and fluoroscopic guidance [16, 17], given the high rate of pneumothoraxes in cases performed without fluoroscopy [18]. Similarly, radial EBUS has been used to visualize vascular structures prior to obtaining the sample, but this approach does not allow for real time guidance during the actual biopsy [19]. Use of a two-scope technique has been employed to re-enter the airway more rapidly in order to visualize and to potentially control bleeding, but does not decrease the incidence of complications [20,21]. Comparable results are found when using prophylactic endobronchial blockers or balloons [22, 23], although the severity of bleeding may decrease. Recent data using vasoconstricting medications and cone-beam computed tomography guidance, however, are promising in reducing complications [12, 24, 25]. Additional studies layering these different modalities together, and in comparison, will be informative to further improve patient safety.

While SLB remains the gold standard approach for obtaining a tissue diagnosis for DPLD, there is an accepted role for TBLC in this process [26]. This value stems from the advantages of TBLC over SLB as a more cost-effective option [3] and its lower complication rates [5, 14, 15]. SLB performed nonelectively on acutely ill-hospitalized patients may lead to a mortality ranging from 6.3 to $16 \%$ [6-9]. In contrast, elective SLB carries a 30-day mortality of $1.5-2.4 \%[9,27,28]$. Our study aimed to investigate whether there was an increased risk of complications among acutely ill-hospitalized patients who underwent TBLC, as is the case for SLB. Our results showed no statistically significant differences in complication rates when performing TBLC for sicker patients (Table 4). Additionally, a final MDC diagnosis was obtained in $95 \%$ of patients undergoing TBLC, suggesting that this procedure may facilitate patient care 
(Table 5). Along with the prior study [10], this data support the safety and utility of TBLC in both inpatients and outpatients.

Final MDC discussion resulted in chronic hypersensitivity pneumonitis $(26 \%, n=20)$ as the most common diagnosis, followed by connective tissue disease-related interstitial lung disease $(17 \%, n=13)$, organizing pneumonia $(9 \%$, $n=7)$, and drug-induced pneumonitis $(7 \%, n=5)$. Few cases of IPF were reported $(5 \%, n=4)$. This finding may be attributable to the increase confidence in radiographic interpretation of chest imaging in determining a definite UIP pattern, thus negating biopsy of these patients, as recommended by guidelines [29]. It is also possible that an increased incidence of pneumothorax may occur when performing TBLC on patients with a definite UIP pattern on imaging given the peripheral honeycombing and subpleural disease, which is the optimal location of the cryoprobe for TBLC. It will be of interest to determine if the incidence of pneumothoraces and diagnosed cases of IPF from TBLC decreases overtime with further refinement in patient selection.

With the development of more data supporting the safety of TBLC on inpatients, it will be important to determine the overall impact of an expedited tissue diagnosis on patient care. Patients with new respiratory symptoms, abnormal PFTs, and/or abnormal chest imaging suggestive of DPLD often wait a prolonged time prior to obtaining a diagnosis and to establishing a treatment plan [30, 31]. The potential impact on patient outcomes, including preservation of lung function as measured by PFTs and lung structure as depicted by HRCT, may be positively impacted by performing TBLC while hospitalized to expedite management and is an area of future study. Additionally, TBLC may play a role in evaluating patients with known DPLD admitted for an exacerbation or concern for disease progression. Understanding if the histology shows a change from an inflammatory state responsive to further immunosuppression to a fibrotic state requiring anti-fibrotic therapy may impact further treatment.

Our study has limitations. These include the smaller sample size of inpatients compared to outpatients and the inability to control for the clinical status, inpatient versus outpatient, of this observational uncontrolled study. However, a strength of our study is its inclusive nature of all patients undergoing TBLC during the study period. We were able to obtain objective data to demonstrate that while inpatients were of higher illness acuity (Tables 1,2 ), TBLC may be a safe procedure with minimal complications (Table 4) and is also effective in obtaining a diagnosis (Table 5).

TBLC is an accepted option for obtaining a tissue sample for patients with DPLD given its comparable accuracy and increased safety profile compared to SLB. Our study suggests that TBLC may be a safe and effective modality in acutely ill-hospitalized patients and outpatients. Our results support considering TBLC in the evaluation of patients who are hospitalized with an acute pulmonary process where a tissue diagnosis may guide management. Efforts to further optimize the procedure to enhance safety and to determine the impact of an expedited tissue diagnosis on patient outcomes are needed.

Author Contributions All authors were responsible for data collection and accuracy of data, analysis of results, finalization of the manuscript, and approval of the submitted article. CC and BSB conceived and wrote the manuscript and took responsibility for the integrity of the work as a whole.

Funding None.

\section{Declarations}

Conflict of interest The authors declare that they have no conflict of interest.

Ethical Approval Approval was obtained from the institutional review board of the Medical College of Wisconsin PRO00036023.

\section{References}

1. Raghu G, Collard HR, Egan JJ et al (2011) An official ATS/ERS/ JRS/ALAT statement: idiopathic pulmonary fibrosis: evidencebased guidelines for diagnosis and management. Am J Respir Crit Care Med 183:788-824

2. Poletti V, Hetzel J (2015) Transbronchial cryobiopsy in diffuse parenchymal lung disease: need for procedural standardization. Respiration 90:275-278

3. Sharp C, McCabe M, Adamali H, Medford, A.R. (2017) Use of transbronchial cryobiopsy in the diagnosis of interstitial lung disease-a systematic review and cost analysis. QJM An International Journal of Medicine, 110: 207-214.

4. Babiak A, Hetzel J, Krishna G et al (2009) Transbronchial cryobiopsy: a new tool for lung biopsies. Respiration 78:203-208

5. Ravaglia C, Bonifazi M, Wells AU et al (2016) Safety and diagnostic yield of transbronchial lung cryobiopsy in diffuse parenchymal lung diseases: a comparative study versus video-assisted thoracoscopic lung biopsy and a systematic review of the literature. Respiration 91(3):215-227

6. Kreider ME, Hansen-Flaschen J, Ahmad NN et al (2007) Complications of video-assisted thoracoscopic lung biopsy in patients with interstitial lung disease. Ann Thorac Surg 83:1140-1145

7. Utz JP, Ryu JH, Douglas WW et al (2001) High short-term mortality following lung biopsy for usual interstitial pneumonia. Eur Respir J 17:175-179

8. Han Q, Luo Q, Xie JX et al (2015) Diagnostic yield and postoperative mortality associated with surgical lung biopsy for evaluation of interstitial lung diseases: A systematic review and meta-analysis. J Thorac Cardiovasc Surg 149(5):1394-1401

9. Hutchinson JP, Fogarty AW, McKeever TM, Hubbard, RB. (2016) In-Hospital Mortality after Surgical Lung Biopsy for Interstitial Lung Disease in the United States. Am J Respir Crit Care Med. 193(10):1161-1167.

10. Cooley J, Balestra R, Aragaki-Nakahodo AA et al (2018) Safety of performing transbronchial lung cryobiopsy on hospitalized patients with interstitial lung disease. Respir Med 140:71-76 
11. Meyer KC, Raghu G, Baughman RP et al (2012) An official American Thoracic Society clinical practice guideline: the clinical utility of bronchoalveolar lavage cellular analysis in interstitial lung disease. Am J Respir Crit Care Med 185(9):1004-1014

12. Benn BS, Romero AO, Bawaadam H, Ivanick N, Lum M, Krishna $\mathrm{G}$ (2021) Cone beam CT guidance improves transbronchial lung cryobiopsy safety. Lung 199(5):485-492

13. Folch EE, Mahajan AK, Oberg CL et al (2020) Standardized definitions of bleeding after transbronchial lung biopsy: a delphi consensus statement from the nashville working group. Chest 158(1):393-400

14. Johannson KA, Marcoux VS, Ronksley PE, Ryerson CJ (2016) Diagnostic yield and complications of transbronchial lung cryobiopsy for interstitial lung disease. A systematic review and metaanalysis. Ann Am Thorac Soc 13(10):1828-1838

15. Iftikhar IH, Alghothani L, Sardi A, Burkowitz D, Musani AI (2017) Transbronchial lung cryobiopsy and video-assisted thoracoscopic lung biopsy in the diagnosis of diffuse parenchymal lung disease. A meta-analysis of diagnostic test accuracy. Ann Am Thorac Soc 14(7):1197-1211

16. Hetzel J, Maldonado F, Ravaglia C et al (2018) Transbronchial cryobiopsies for the diagnosis of diffuse parenchymal lung diseases: expert statement from the cryobiopsy working group on safety and utility and a call for standardization of the procedure. Respiration 95(3):188-200

17. Maldonado F, Danoff SK, Wells AU et al (2020) Transbronchial cryobiopsy for the diagnosis of interstitial lung diseases: CHEST Guideline and Expert Panel Report. Chest 157(4):1030-1042

18. DiBardino DM, Haas AR, Lanfranco AR, Litzky L, Sterman D, Bessich J (2017) High complication rate after introduction of transbronchial cryobiopsy into clinical practice at an academic medical center. Ann Am Thorac Soc 14:851-857

19. Berim IG, Saeed AI, Awab A, Highley A, Colanta A, Cahundry F (2017) Radial probe ultrasound-guided cryobiopsy. J Bronchology Interv Pulmonol 24:170-173

20. Sriprasart T, Aragaki A, Baughman R et al (2017) A single US center experience of transbronchial lung cryobiopsy for diagnosing interstitial lung disease with a 2-scope technique. J Bronchology Interv Pulmonol 24:131-135

21. Bango-Álvarez A, Ariza-Prota M, Torres-Rivas H et al (2017) Transbronchial cryobiopsy in interstitial lung disease: experience in 106 cases - how to do it. ERJ Open Res 3(1):00148-02016
22. Dhooria S, Mehta RM, Srinivasan A et al (2018) The safety and efficacy of different methods for obtaining transbronchial lung cryobiopsy in diffuse lung diseases. Clin Respir J 12(4):1711-1720

23. Pajares Ruiz V, Torrego Fernández A, Puzo Ardanuy C, Gil de Bernabe A. Use of an occlusion balloon in transbronchial lung cryobiopsy. Arch Bronconeumol. 2014 ;50(7):309-310.

24. Steinfort DP, D’Agostino RD, Vrjlic I et al (2018) CT-fluoroscopic guidance for performance of targeted transbronchial cryobiopsy: a preliminary report. Respiration 96(5):472-479

25. Zhou G, Ren Y, Li J et al (2020) Safety and diagnostic efficacy of cone beam computed tomography-guided transbronchial cryobiopsy for interstitial lung disease: a cohort study. Eur Respir J 56(2):2000724

26. Hariri LP, Roden AC, Chung JH et al (2021) The role of surgical lung biopsy in the diagnosis of fibrotic interstitial lung disease: perspective from the pulmonary fibrosis foundation. Ann Am Thorac Soc 18(10):1601-1609

27. Hutchinson JP, McKeever TM, Fogarty AW, Navaratnam V, Hubbard R (2016) Surgical lung biopsy for the diagnosis of interstitial lung disease in England: 1997-2008. Eur Respir J 48(5):1453-1461

28. Nguyen W, Meyer KC (2013) Surgical lung biopsy for the diagnosis of interstitial lung disease: a review of the literature and recommendations for optimizing safety and efficacy. Sarcoidosis Vasc Diffuse Lung Dis 30(1):3-16

29. Raghu G, Remy-Jardin M, Myers JL, et al. Diagnosis of Idiopathic Pulmonary Fibrosis. An Official ATS/ERS/JRS/ALAT Clinical Practice Guideline. Am J Respir Crit Care Med. 2018;198(5):e44-e68.

30. Pritchard D, Adegunsoye A, Lafond E et al (2019) Diagnostic test interpretation and referral delay in patients with interstitial lung disease. Respir Res 20(1):253

31. Collard HR, Tino G, Noble PW et al (2007) Patient experiences with pulmonary fibrosis. Respir Med 101(6):1350-1354

Publisher's Note Springer Nature remains neutral with regard to jurisdictional claims in published maps and institutional affiliations. 